\title{
Digital Dental Academy in Berlin eröffnet
}

Die digitale Zahnheilkunde wird seit Jahrzehnten durch CEREC geprägt. Um den Zahnärzten eine optimale Fortbildung zu ermöglichen, haben führende Mitglieder der Deutschen Gesellschaft für computergestützte Zahnheilkunde (DGCZ) in Berlin die Digital Dental Academy (DDA) gegründet. Sie wurde im Juli feierlich eröffnet.

Dentsply Sirona CAD/CAM hat das Trainingszentrum maßgeblich gefördert und ausgestattet. Um den Zahnärzten in ansprechender Umgebung mit optimaler didaktischer Ausstattung Fortbildungskurse zu CAD/CAM-Anwendungen anbieten zu können, haben sich 13 führende Vertreter der DGCZ sowie Zahnärzte und Professoren zusammengeschlossen und mit der Digital Dental Academy ein CERECTrainingszentrum gegründet. Einer von ihnen ist Dr. Klaus Wiedhahn, ein erfahrender CEREC-Anwender und -Trainer: „Wir möchten zum einen unsere Begeisterung für CAD/CAM in der Zahnarztpraxis weitergeben und zum anderen mit diesem Angebot das weiterführende Kursgeschehen beleben.“

Die Academy am Katharina-HeinrothUfer in Berlin wurde im Beisein des CEREC-Entwicklers Prof. Dr. Dr. Werner Mörmann feierlich eröffnet. Rund 80 Zahnärzte und Medienvertreter gehörten zu den Ersten, die die Räumlichkeiten kennenlernen und an einem exklusiven CEREC-Training teilnehmen durften.

Nach einer Pressemitteilung von Dentsplv Sirona CAD/CAM, USA - York 